\title{
A SIMPLE EXAMPLE OF A NON-COMMUTATIVE ARENS PRODUCT
}

\author{
IGNACIO ZALDUENDO
}

\begin{abstract}
A simple and natural example is given of a non-commuting Arens multiplication.
\end{abstract}

In [1] and [2], R. Arens described a way to extend the product of an associative Banach algebra $A$ to its bidual $A^{* *}$ which renders it a Banach algebra. Indeed, there are two such extensions, both equally canonical.

It was proved by M. Day [5] that $A^{* *}$ need not be commutative, even if $A$ is. Civin and Yood [4] also gave various conditions guaranteeing noncommutativity of $A^{* *}$ in several cases. In [6], J. Rennison produced a simple example of a commutative Banach algebra whose bidual is not commutative. This ingenious example has, however, the appearance of artificiality. The purpose of the present note is to give an example which is both trivial and more natural.

It is certainly well known, that the example given below is non-commutative, as it is a special case of the general results found in [4] and [5]. The point, however, is that this can be proven directly after giving the definition of Arens product. This is what we now proceed to do.

Definition of the Arens product. Let $A$ be a Banach algebra. In what follows, $a$ and $b$ will denote elements of $A, \gamma$ an element of $A^{*}, S$ and $T$ elements of $A^{* *}$. Define $a \gamma$ and $T_{\gamma}$, elements of $A^{*}$, by

$$
(a \gamma)(b)=\gamma(a b)
$$

and

$$
T_{\gamma}(\alpha)=T(a \gamma)
$$

Now define $S . T \in A^{* *}$, by

$$
(S . T)(\gamma)=S\left(T_{\gamma}\right)
$$


$(S, T) \longmapsto S . T$ is one of the two possible Arens products, when $A$ is commutative; The other is simply $(S, T) \longmapsto T . S$, which need not be the same, as the following example shows.

The example. We shall take $A=l^{1}(\mathbb{Z})$, with the usual Banach algebra structure. Note that its dual $A^{*}$ is $l^{\infty}(\mathbb{Z})=C(\beta \mathbb{Z})$; and its bidual $A^{* *}$ is the space of regular complex Borel measures on the Stone-Cech compactification $\beta \mathbb{Z}$ of $\mathbb{Z}$. It is easy to check that

$$
(a \gamma)_{n}=\Sigma a_{k} \gamma_{n+k}
$$

Define the following subspace of $A^{*}$.

$$
V=\left\{\gamma \in A^{*}: L^{-}(\gamma)=\lim _{k \longrightarrow \infty} \gamma_{k}, \text { and } L^{+}(\gamma)=\lim _{k \longrightarrow \infty} \gamma_{k} \text { exist }\right\}
$$

Now extend the bounded linear functionals $L^{-}$and $L^{+}$to $A^{*}$ by Hahn-Banach, and denote these extensions $S^{-}$and $S^{+}$. Let $\gamma \in A^{*}$ be

$$
\gamma=(\ldots,-1,-1,-1,0,1,1,1, \ldots), \text { where } 0=\gamma_{0}
$$

Then

$$
S_{\gamma}^{-}(a)=S^{-}(a \gamma)=\lim _{n \rightarrow \infty}\left(-\sum_{k<-n} a_{k}+\sum_{k>n} a_{k}\right)=-\sum_{k} a_{k}
$$

Hence $S_{\gamma}^{-}$is the element of $A^{*}$

$$
S_{\gamma}^{-}=(\ldots,-1,-1,-1, \ldots)
$$

In a completely analogous manner, one sees that

$$
S_{\gamma}^{+}=(\ldots, 1,1,1, \ldots)
$$

Therefore,

$$
\left.\left(S^{+} . S^{-}\right)(\gamma)=S^{+}\left(S_{\gamma}^{-}\right)=-1 \neq 1=S^{-}\left(S_{\gamma}^{+}\right)=S^{-} . S^{+}\right)(\gamma)
$$

and $A^{* *}$ is not commutative.

The Arens product in $A^{* *}$ extends the notion of convolution of measures. For more on Arens products and measures, see [7] and [8]. 


\section{References}

1. R. ARENS, Operations induced in function classes, Monatsh. Math. $\mathbf{5 5}$ (1951), 1-19.

2. R. ARENS, The adjoint of a bilinear operation, Proc. AMS 2 (1951), 839-848.

3. P. Civin, Extensions of homomorphisms, Pacific J. of Math. 11 (1961), 1223-1233.

4. P. CIVIN AND B. YOOD, The second conjugate space of a Banach algebra as an algebra, Pacific J. of Math. 11 (1961), 847-870.

5. M. DAY, Amenable semigroups, Ml. J. of Math. 1 (1957), 509-544.

6. J. Rennison, A note on the extension of associative products, Proc. AMS 17 (1966), 1375-1377.

7. J. RENNison, Arens products and measure algebras, LMS 44 (1969), 369-377.

8. J. RENNISON, Arens products and measure algebras-a supplement, LMS (2) $1(1969), 232-236$.

Instituto Argentino de Matemática

Viamonte $16362^{9}$ piso

1055 Buenos Aires

ARGENTINA

Rebut el 20 d'Agost de 1990 